\title{
Detection of Extended - Spectrum Beta - Lactamases in Escherichia coli Isolates and it's Correlation with Vesicoureteral Reflux Nephropathy
}

\author{
Parsa Yousefichaijan, ${ }^{1}$ Masoud Rezagholizamenjany, ${ }^{2,}{ }^{*}$ Fatemeh Safi, ${ }^{3}$ Fatemeh Rafiei, ${ }^{4}$ and Ali \\ Arjmand $^{5}$ \\ ${ }^{1}$ Department of Pediatric Nephrology, Amir Kabir Hospital, Arak University of Medical Sciences, Arak, Iran \\ ${ }^{2}$ Arak University of Medical Sciences, Arak, Iran \\ ${ }^{3}$ Department of Radiology, Arak University of Medical Sciences, Arak, Iran \\ ${ }^{4}$ Department of Biostatistics, Endocrinology and Metabolism Research Center, Arak University of Medical Sciences, Arak, Iran \\ ${ }^{5}$ Department of Pediatric, Amir Kabir Hospital, Arak University of Medical Sciences, Arak, Iran \\ "Corresponding author: Masoud Rezagholizamenjany, Medical Students, Arak University of Medical Sciences, Arak, Iran. Tel: +98-9184374727, E-mail: \\ masoudrezagholi074@gmail.com
}

Received 2017 April 25; Revised 2017 June 19; Accepted 2017 August 28

\begin{abstract}
Background: Vesicourethral reflux is the reverse flow of urine from the bladder to the ureter and then to kidneys. $30 \%$ to $40 \%$ of children are infected with urinary tract infections disease, and recenthly, one of the most common urinary tract infections has become Extended - Spectrum Beta - Lactamases (ESBL) Escherichia coli isolates.

Objectives: The aim of this was to investigate the correlation between extended Extended Spectrum Beta Lactamase Escherichia coli isolates and reflux nephropathy disease in children.

Methods: This was a case - cotrol study conducted on 200 children with or without reflux nephropathy referred to the pediatric clinic. The researchers selected two groups of children; 100 children with reflux nephropathy and 100 healthy children. Urinary tract infection was tested by urine culture and antibiogram. Correlation of reflux nephropathy and ESBL Escherichia coli infection were analyzed by the SPSS program.

Results: Infected children with Extended Spectrum Beta Lactamase E. coli infection compared with healthy children, were more influenced by reflux nephropathy. Overall, 53\% of case and 9\% of control group had infection of ESBL Escherichia coli.

Conclusions: Reflux nephropathy can be controlled by prevention of these types of infections.
\end{abstract}

Keywords: Reflux Nephropathy, Extended Spectrum Beta Lactamase, E. coli, Children

\section{Background}

Vesicoureteral reflux refers to reverse flow of the urine from the bladder to the ureter and then to kidneys $(1,2)$. In a normal position, the ureteral attachment to the bladder is oblique, and creates a flap - valve mechanism and prevents reflux (3-5). However, reflux occurs when the sub mucosal tunnel between the mucosa and detrusor muscle is short or absent; in other words VUR mostly results from congenital inefficiency of the Ureterovesical (UV) junction $(1,2)$.

Reflux predisposes the person to infection of the kidneys, by facilitating the transport of bacteria from the bladder and lower urinary tract to the kidneys and upper urinary tract (6). When infection ascend the kidneys, pyelonephritis and inflammatory reaction could occur that result in kidney injury and scarring, which is also called reflux nephropathy or reflux - related renal injury. In addition, reflux nephropathy is a great global public health problem, the incidence of which in the world is steadily increasing amongst children $(7,8)$. Although febrile UTI at any age leads to pyelonephritis and then renal scarring, yet risk of UTI is highest in children younger than two years of age (9-11). Patients with febrile urinary tract infection and VUR were three times more likely to develop kidney injury compared to those without reflux $(1,7)$.

Urinary tract infection is one of the most common childhood infections that may be associated with vesicoureteral reflux (12). The infection may be symptomatic or asymptomatic; asymptomatic is colonization without any symptoms while symptomatic infection is concurrent with microbial colonization symptoms $(3,13)$. Infection prevalence is greater in children and females $(7,14)$. In addition, gram - negative bacilli are the most common infections that lead to urinary tract infections; E. coli has caused $80 \%$ of these infections, and ESBL - positive lead to more mortality and morbidity in children (15). Therefore, reflux accompanied with urinary tract infection lead to increased risk of renal injury and scarring (16). 


\section{Objectives}

Urinary Tract Infections, as a high prevalence disorder, especially among females, can cause nephropathy. The aim of this study was to investigate children with kidney reflux nephropathy and its correlation with infection of ESBL $E$. coli bacteria in Arak city.

\section{Methods}

\subsection{Inclusion and Exclusion Criteria}

Inclusion criteria were absence of congenital kidney disease and consent to participate in the research project, age of one month to 12 years old, male and female gender. The exclusion criteria were presence of congenital renal abnormality, incomplete study process, and patient's lack of satisfaction with using their personal data in the study.

\subsection{Study Setting}

This was a case control and hospital - based study, which was conducted in Amir Kabir Hospital of Arak city.

\subsection{Study Population}

Samples was taken based on other studies, and the researchers considered 200 children for study conduction and patients were followed for 12 months (4). From 200 children, 100 children with reflux nephropathy (grade IV and V of VUR) as the case group, and 100 healthy children, without any disease, as the control group (control group selected from outpatients that were not affected by severe or systemic disease) were considered.

\subsection{Measurements}

At first, Voiding Cystourethrogram (VCUG) was performed on all children of case and control groups, while dimercaptosuccinic acid (DMSA) scan was performed on patients with positive VUR diagnosis, four to six months after acute UTI. Infection of ESBL E. coli bacteria was detected by urine culture, with standard loop in hospital admission, and resistance to various antibiotics was determined based on anti - biogram test, Disk Agar Diffusion approach, and patients were treated with usual antibiotics (Ceftriaxone at a dose of 50 to $75 \mathrm{mg} / \mathrm{kg} /$ day/iv in hospitalized patients and cefixime $8 \mathrm{mg} / \mathrm{kg} /$ day/po in outpatients). Moreover, demographic characteristics of patients including age, gender, education of parents, place of residence and maternal age in pregnancy was reviewed by interviews of doctors with patients.

\subsection{Ethical Considerations}

Ethical issues (including plagiarism, data fabrication, and double publication) were completely observed by the authors. In addition, the ethical committee of Arak University of Medical Sciences approved the study protocol with the ethical code of IR.ARAKMU.REC.1394.344.

\subsection{Statistical Analysis}

Data analysis was conducted by the chi-square test and significance level was considered as $\mathrm{P}<0.05$. The correlation of VUR nephropathy and infection of ESBL E. coli was evaluated.

\section{Results}

Table 1 shows the demographic information, and based on this demographic information, there were statistically significant differences regarding age, gender, parents education, living area, and maternal age of pregnancy $(\mathrm{P}=$ 0001). In addition, as shown in Table 2, ESBL E. coli infection in the case group was significantly higher than the control group $(\mathrm{P}=0001)$.

\begin{tabular}{|c|c|c|c|}
\hline Variables $^{\mathrm{a}}$ & Case & Control & P Value \\
\hline Age $^{\text {b }}$ & $12 \pm 3$ & $48 \pm 2$ & 0.0001 \\
\hline Gender & & & 0.0001 \\
\hline Male & 13 & 7 & \\
\hline Female & 87 & 93 & \\
\hline Parents education & & & 0.0001 \\
\hline Diploma and lower & 4 & 20 & \\
\hline Bachelor & 89 & 67 & \\
\hline Master's degree and higher & 7 & 13 & \\
\hline Living place & & & 0.0001 \\
\hline Urban & 68 & 91 & \\
\hline Rural & 32 & 9 & \\
\hline Pregnancy maternal age $^{c}$ & & & 0.0001 \\
\hline$<20$ & 15 & 10 & \\
\hline $21-30$ & 15 & 81 & \\
\hline $31-40$ & 61 & 4 & \\
\hline$>41$ & 9 & 5 & \\
\hline
\end{tabular}

${ }^{\mathrm{a}}$ Presented as percentage

${ }^{\mathrm{b}}$ Presented as mean \pm SD and month

${ }^{\mathrm{c}}$ Years old

In Table 3 resistance to various antibiotics in VUR nephropathy was determined, and it was observed that 
Table 2. ESBL E. coli Infection Information's in Case (n: 100) and Control (n: 100) Groups

\begin{tabular}{|c|c|c|c|}
\hline ESBL E. coli Infection ${ }^{a}$ & Case & Control & P Value \\
\hline Positive & 53 & 9 & 0.0001 \\
\hline Negative & 47 & 91 & \\
\hline
\end{tabular}

${ }^{\mathrm{a}}$ Presented as percentage

there was resistance to multiple types of antibiotics so that the greatest resistance was for cefixime, ampicillin and cephalothin (100\%) and ofloxacin (29\%) and nitrofurantoin resistance was lowest.

\begin{tabular}{|lc|}
\hline Table 3. Antibiotics Resistance in VUR Nephropathy Children \\
\hline Antibiotics & Resistance Percentage \\
\hline Cefotaxime & 98 \\
\hline Cefixime & 100 \\
\hline Ceftriaxone & 99 \\
\hline Gentamicin & 73 \\
\hline Amikacin & 69 \\
\hline Ampicillin & 100 \\
\hline Cotrimoxazole & 89 \\
\hline Amoxiclav & 69 \\
\hline Nitrofurantoin & 39 \\
\hline Ciprofloxacin & 41 \\
\hline Ofloxacin & 29 \\
\hline Cephalothin & 100 \\
\hline Nalidixic acid & 57 \\
\hline Ceftazidime & 95 \\
\hline
\end{tabular}

\section{Discussion}

Infection can influence VUR and lead to increased risk of nephropathy. Furthermore, ESBL E. coli bacteria, as an important microorganism, has multiple drug resistance. In the current study, the correlation of ESBL E. coli infection and reflux nephropathy was proved, yet this correlation was not investigated in numerous other studies.

Sweeney et al. in a study about reflux nephropathy in children with UTIs, evaluated 81 male and 46 female infants. They concluded that incidence of VUR remained lower than cases with urinary tract infection (17). Chih et al., in a study about nephropathy in infants with urinary tract infection, evaluated 152 young infants ( 127 males and 25 females) and concluded that $E$. coli was the most common pathogen in urinary tract infection and had high ampicillin resistance, and leukocytosis indicated the risk of VUR while fever indicated a risk of pyelonephritis (18). Koshesh et al., in their study, evaluated ESBL E. coli infections and they found that these isolates were higher in communities rather than inpatients (19). Rahman et al. in a case - control study reported male gender and high grade reflux as risk factors of nephropathy among children with UTI; also, the current study demonstrated that reflux was seen as a risk factor for urinary tract infections (7). In another study, Ramalingam et al. found that voiding dysfunction was considerably higher in females compared to males and voiding dysfunction was higher among those with first episode of UTI (3). Mishra et al. concluded that UTI in geriatric patients could result in significant complications; they found UTI in $28.5 \%$ of geriatric patients and E. coli was the most frequent pathogen (16). Yousefichaijan et al. showed a certain association between hyponatremia and reflux nephropathy (20). Ranjbar et al., in their study, evaluated molecular epidemiology of Klebsiella pneumonia and found that blaCTX in K. pneumoniae isolates was at an alerting rate (21). In another study on risk factors of infantile reflux expressed in subgroup analysis by CKD subtype, low birth weight and maternal pregestational DM were considerable along with an increased risk of renal dysplasia/aplasia while low birth weight, maternal gestational DM, and maternal overweight/obesity were significantly associated with obstructive uropathy (22). Fallah et al. in their study found that Enteropathogenic Escherichia coli was a major cause of diarrhea in Iranian children (23). However, patients with congenital disorders were not included in the current study.

Furthermore, in a study by Yousefichaijan et al. in 2014, they concluded that there is an obvious solidarity between reflux nephropathy and mean platelet volume (24). Also, in another study by the same authors, it was concluded that percentage of sacral ratio abnormality in children with VUR was more than children without this (10). Therefore, it could be suggested that it is essential to identify and treat voiding dysfunction in children with UTI and the current study confirms these findings. The limitation of the current study was the small sample size in the two groups and lack of cooperation by some patients and their parents. Accordingly, further studies, especially prospective ones with greater number of patients, will be needed in the future to evaluate infection of ESBL E. coli in children with vesicoureteral reflux nephropathy.

\subsection{Conclusion}

Correlation between ESBL E. coli infection and VUR (grade IV and V) nephropathy was approved. In addition, the importance of drug resistance of UTI in VUR nephropathy has been approved. The best antibiotic for this infection was ofloxacin and nitrofurantoin. Therefore, early di- 
agnosis and treatment of UTI, especially of ESBL E. coli infection can prevent serious complications and lead to healthy outcomes.

\section{Acknowledgments}

The authors gratefully acknowledge the Research Council of Arak University of Medical Sciences (Grant Number: 2481) for their financial support.

\section{Footnote}

Funding/Support: The current study was funded by Arak University of Medical Sciences.

\section{References}

1. Hodson CJ, Maling TM, McManamon PJ, Lewis MG. The pathogenesis of reflux nephropathy (chronic atrophic pyelonephritis). Br J Radiol. 1975;Suppl 13:1-26. [PubMed: 766885]

2. Tokhmafshan F, Brophy PD, Gbadegesin RA, Gupta IR. Vesicoureteral reflux and the extracellular matrix connection. Pediatr Nephrol. 2017;32(4):565-76. doi: 10.1007/s00467-016-3386-5. [PubMed: 27139901]. [PubMed Central: PMC5376290].

3. Ramalingam R, James S, Babu R, Rajendran P. Voiding dysfunction in children with culture positive urinary tract infection. Int J Contemp Pediatr. 2015:375-8. doi: 10.18203/2349-3291.ijcp20150978.

4. Cyrus A, Dorreh F, Sharafkhah M, Safi F, Naziri M, Taherahmadi H. Correlation of Sacral Ratio and Reflux-Related Renal Injury in Children with Vesicoureteral reflux with and without Nephropathy. J Pediatr Nephrol. 2014;2(3):116-8.

5. Yousefichaijan P, Rezagholizamenjany M, Rafiei F, Taherahmadi $\mathrm{H}$ Rafiei M. The relationship between blood biomarkers level and the prognosis of nephrotic syndrome in the children. Int J Pediatr. 2016;4(9):3489-97.

6. Yousefichaijan P, Dorreh F, Rafiei M, Naziri M. Effective factors in growth and development in children and infants with vesicoureteral reflux (VUR). Med J Mashhad Univ Med Sci. 2014;57(5):690-6.

7. Rahman H, Al Mamun A, Roy RR, Haque SS, Muinuddin G. Screening for Vesico ureteral Reflux and Renal Scar in Patients Presented with Urinary Tract Infection. J Pediatr Nephrol. 2015;3(3):95-9.

8. Nino F, Ilari M, Noviello C, Santoro L, Ratsch IM, Martino A, et al. Genetics of Vesicoureteral Reflux. Curr Genomics. 2016;17(1):70-9. doi: 10.2174/1389202916666151014223507. [PubMed: 27013925]. [PubMed Central: PMC4780477].

9. Yousefi P, Cyrus A, Moghaddasi Z, Dorreh F, Aravand A. The frequency of recurrence of urinary tract infection (UTI) in 1-month to 12-yearold children without congenital abnormalities referred to Arak Amir Kabir Hospital. Zahedan Univ Med Sci J. 2011;19(76):66-76.
10. Macedo M, Martins JL, Freitas Filho LG. Sacral ratio and fecal continence in children with anorectal malformations. BJU Int 2004;94(6):893-4. doi: 10.1111/j.1464-410X.2004.05053.x. [PubMed 15476529].

11. Rezagholi-Zamnjany M, Yousefichaijan P. An overview on peritoneal dialysis. Ann Res Dial. 2016;1(1).

12. Kumar R, Dayal D, Gupta A, Kumar R, Sodhi KS, Bhattacharya A Acute Focal Bacterial Nephritis Secondary to Vesicoureteric Reflux in a Young Boy. Arc Pediatr Infect Dis. 2015;3(4). doi: 10.5812/pedinfect.26552.

13. Doern CD, Richardson SE. Diagnosis of Urinary Tract Infections in Children. J Clin Microbiol. 2016;54(9):2233-42. doi: 10.1128/JCM.0018916. [PubMed: 27053673]. [PubMed Central: PMC5005475].

14. Geback C, Hansson S, Martinell J, Sandberg T, Jodal U. Urinary tract infection pattern in adult women followed from childhood. Pediatr Nephrol. 2016;31(7):1107-11. doi: 10.1007/s00467-016-3342-4. [PubMed: 26879801].

15. Hasani A, Purmohammad A, Ahangarzadeh Rezaee M, Hasani A, Dadashi M. Integron-Mediated Multidrug and Quinolone Resistance in Extended-Spectrum $\beta$-Lactamase-Producing Escherichia coli and Klebsiella pneumoniae. Arc Pediatr Infect Dis. 2017;5(2)

16. Mishra N, Tripathi SM, Jain M, Mishra R, Patel SS, Singh MK. Bacteriological study of urinary tract infection in geriatric patients. J Clin Microbiol. 2016:255-64.

17. Sweeney B, Cascio S, Velayudham M, Puri P. Reflux nephropathy in infancy: a comparison of infants presenting with and without urinary tract infection. J Urol. 2001;166(2):648-50. [PubMed: 11458111].

18. Yen C-W, Hung K-L. Pyelonephritis and Reflux Nephropathy in Young Infants with Urinary Tract Infection. JAMA. 2005;3(4):221-9.

19. Koshesh M, Mansouri S, Hashemizadeh Z, Kalantar-Neyestanaki D. Identification of extended-spectrum $\beta$-lactamase genes and ampc- $\beta$ lactamase in clinical isolates of escherichia coli recovered from patients with urinary tract infections in Kerman, Iran. Arc Pediatr Infect Dis. 2017;5(2).

20. Yousefichaijan P, Taherahmadi H, Rafiei M, Shariatmadari F, Alinejad S, Ghandi Y, et al. The association between hyponatremia and reflux-related renal injury in acute pyelonephritis. J Pediatr Nephrol. 2015;3(3):104-8.

21. Ranjbar R, Memariani H, Sorouri R. Molecular Epidemiology of Extended-Spectrum Beta-Lactamase-Producing Klebsiella pneumoniae Strains Isolated from Children with Urinary Tract Infections. Arc Pediatr Infect Dis. 2017;5(2).

22. Yousefichaijan P, Safi F, Rafiei M, Taherahmadi H, Fatahibayat GA Naziri M. Prenatal Risk Factors for Infantile Reflux Nephropathy. J Pediatr Nephrol. 2015;3(4):135-8.

23. Mohammadzadeh M, Goudarzi H, Dabiri H, Fallah F. Characterization of Enteropathogenic Escherichia coli Associated with Diarrhea Among Iranian Infants. Arc Pediatr Infect Dis. 2017;5(1).

24. Yousefichaijan P, Rafiei M, Eghbali A, Sharafkhah M, Taherahmad $\mathrm{H}$, Naziri M, et al. Mean platelet volume: a useful marker in reflux nephropathy. J Pediatr Nephrol. 2014;2(4):137-9. 